\title{
Inhalt
}

\section{Therapie der Hyperthyreose}

\subsection{Neues zur Pathogenese des Morbus Basedow}

Neues zur Pathogenese des Morbus Basedow: der TSH-Rezeptor

M. Derwahl

Alternative splicing of the human TSH receptor: a possible role of the soluble TSH receptor in the aetiology of Graves' disease

N. Hunt, K. P. Willey

TSH-Rezeptorantikörper beim Morbus Basedow

R. Hoermann

Postpartum-Hyperthyreose: Sind positive TSH-Rezeptorantikörper für M. Basedow beweisend?

A. Kurtaran, J. Flores, M. Weissel

Immunmechanismen beim Morbus Basedow

A. E. Heufelder

Möglicher Transfer einer immunogenen Hyperthyreose bei einer Knochenmarktransplantation (KMT)

S. Lederbogen, K. Quabeck, H. Grosse-Wilde, K. Badenhoop,

K. H. Usadel, Th. Olbricht, D. Reinwein

Schilddrüsenantikörper und die Intensität des intrathyreoidalen Autoimmunprozesses bei Morbus Basedow

R. Paschke

Neues zur Pathogenese des Morbus Basedow: Zusammenfassung und Aspekte für die zukünftige Therapie

K. Mann

1.2 Morbus Basedow und Autonomie

Hyperthyreosetherapie: state of the art

D. Reinwein

Konservative Therapie des Morbus Basedow 
VIII Inhalt

Pharmakokinetik von Methimazol und seinem Metaboliten

3-Methyl-2-Thiohydantoin

D. Aktuna, W. Langsteger, W. Buchinger, W. Florian, B. Schubert,

K. Dominik, O. Eber

Changes of level of thyroid autoantibodies (TBII, TSab, anti-HTG, anti-TPO) during methimazole treatment of patients with Basedow's disease

Gy. Bakó, E. Nagy, E. Fórizs, Zs. Karányi, E. Mezősi, A. Leövey

Langzeit-follow-up von 196 Morbus-Basedow-Patienten nach initial unterschiedlich hoher (10 versus $40 \mathrm{mg}$ ) Methimazol-Therapie

A. Rawert, G. Benker, D. Reinwein

Definitive Therapie bei Morbus Basedow

W. Meng

Radiojodtherapie des M. Basedow und der funktionellen Autonomie: state of the art

Chr. Reiners

Entwicklung einer immunogenen Hyperthyreose

nach Radiojodtherapie einer (uni-)fokalen Schilddrüsenautonomie

B. Boddenberg, E. Voth, H. Schicha

Ergebnisse der Radiojodtherapie bei fokaler Schilddrüsenautonomie und Immunthyreopathie M. Basedow

C. A. Guhlmann, J. Rendl, P. Kornecki, W. Börner

Bedeutung des Suppressionsuptakes (TcTUs) zur besseren Abschätzung der Aktivität für die Radiojodtherapie bei verschiedenen

Erscheinungsformen der funktionellen Autonomie der Schilddrüse

M. Reinhardt, H. Blattmann, D. Emrich, E. Moser

Conclusio

M. Hüfner

Plummern bei Morbus Basedow

Th. Olbricht, K. W. Sievers, U. Krause

Operationsindikation und chirurgisches Vorgehen bei Hyperthyreose

A. Frilling, A. Stenger, E. Achilles

Chirurgie der Hyperthyreose bei Morbus Basedow:

Hemithyreoidektomie versus subtotale Resektion

B. Bein, S. Wähling, F. Spelsberg 
Inhalt IX

Immunogene Hyperthyreose und funktionelle Autonomie

$J$. Friedrich, T. Olbricht, M. Luster, U. Krause

Effektivität der chirurgischen Therapie in der Behandlung der

Hyperthyreose

I. Gál, D. Nagy, S. Saortay, E. Kosa

Simultanoperationen an Herz und Schilddrüse

U. Krause, U. Wolfhard, T. Olbricht, M. K. Walz, S. Müller

172

Bedeutung des Volumens der Restschilddrüse für die Entwicklung eines Hyperthyreoserezidivs nach operativer Therapie der

Immunthyreopathie vom Typ M. Basedow

E. Werner, J. Rendl, W. Börner

Postoperative Schilddrüsenfunktion nach chirurgischer Therapie bei

Morbus Basedow

J. Witte, P. E. Goretzki, R. Carius, H. D. Röher

Operationstaktik bei chirurgischer Hyperthyreosebehandlung

T. Setschanov, R. Pandev, I. Mendisov, G. Gantschev

Conclusio

H. Dralle

1.3 Unkonventionelles therapeutisches Vorgehen

Unkonventionelles therapeutisches Vorgehen bei Hyperthyreose Blick über den Zaun: Therapie außerhalb Deutschlands

Th. Olbricht

Von Pflanzenextrakten zu Hormonantagonisten - neue experimentelle

Konzepte zur Hyperthyreosetherapie

J. Köhrle

Autonomous thyroid nodule and percutaneous ethanol injection:

was it love at first sight?

L. Baschieri, F. Monzani, N. Caraccio, P. V. Lippolis, A. Casolaro,

O. Goletti

Sonographisch gezielte perkutane Alkoholinstillation in der Therapie dekompensierter autonomer Schilddrüsenadenome

W. Blank, B. Braun

Behandlung autonomer Schilddrüsenadenome durch intranoduläre

Äthanolinjektionen unter Ultraschallkontrolle

$K$. W. Wenzel

Conclusio

D. Emrich 
X Inhalt

\subsection{Hyperthyreose im Kindesalter}

Diagnose und Behandlung der Hyperthyreose im Kindesalter:

Ergebnisse einer Umfrage in Europa

H. Perrild

Besonderheiten der Therapie der Hyperthyreose im Kindesalter

F. Péter

Spezielle Aspekte für die chirurgische Therapie der Hyperthyreose im Kindesalter

J. Witte, P. E. Goretzki, A. Müller, H. D. Röher

Neonatale Hyperthyreose bei unbehandeltem maternalen M. Basedow

- ein Fallbericht mit Literaturvergleich

C. Krüger, H. G. Dörr, M. Zant, C. Kändler, R. Beyer, W. Becker,

F. Wolf, D. Harms

\subsection{Seltene Formen der Hyperthyreose}

Hyperthyreose und Thyreoiditis

U. Bogner

Inappropriate TSH-Sekretion

G. Brabant, K. Ocran

Hyperthyreose bei Trophoblastentumoren

B. Saller

Seltene Formen der Hyperthyreose: Struma ovarii

A. Kroiss

Jodinduzierte Hyperthyreose

P. M. Schumm-Draeger

Jodidtherapie bei komplizierter Thyreotoxikose

E. Kallee, D. Luft, J. Saal, B. Steinke, R. Wahl

Schilddrüsenantikörper vor und nach Plummerung bei Patienten mit Morbus Basedow

W. Reinhardt, Th. Olbricht, F. Jockenhövel, K. Cissewski,

$S$. Lederbogen, U. Krause, D. Reinwein

Seltene Hyperthyreoseformen: Interferon-induzierte Thyreopathien 
Hyperthyreose vom Typ M. Basedow bei primärer

Nebennierenrindeninsuffizienz

M. Ventz, M. Lehsnau, G. Knappe, H. Gerl, R. Michael

Zusammenfassung

K. W. Wenzel

\subsection{Schilddrüse und Knochen}

Schilddrüsenhormone und Knochenstoffwechsel

M. Peterlik

Schilddrüsenhormone und Osteoporose

Ch. Wüster

Schilddrüsenhormongabe - ein Risikofaktor für spätere Osteoporose? 395 H.-Chr. Schober

2 Neues zur Diagnostik und Therapie der Schilddrüsenerkrankungen

\subsection{Diagnostik}

Messung der Jodidkonzentration im Urin mit „Paired-ion, reversedphase high-performance liquid chromatography“ und elektrochemischer Detektion

J. Rendl, S. Seybold, W. Börner

Erste Ergebnisse und Methoden zum Nachweis von AntifibroblastenAntikörpern (aFA) bei Patienten mit endokriner Ophthalmopathie P. Scheibner, F. Tatzber, K. Müllner, P. Pfragner, H. Esterbauer, E. Brunner, O. Eber

Entwicklung eines Festphasenenzymimmunoassays zur Bestimmung von Autoantikörpern gegen die Schilddrüsenhormone $\mathrm{T}_{3}$ und $\mathrm{T}_{4}$ H.-J. Knopf, C. Zahn, G. Müller

Serum-Thyreoglobulin bei hospitalisierten chronischen geriatrischen Patienten: eine Screening-Studie

I. Szabolcs, W. Bernard, F. A. Horster

Thiocyanat im Serum bei euthyreoter Struma, Hyper- und Hypothyreose

Th. Olbricht, U. Krause, S. Lederbogen, D. Reinwein 
XII Inhalt

Farbcodierte Doppler-Sonographie zur Differenzierung der Hyperthyreose

B. Braun, W. Blank, P. Gelinsky

Bestimmung des funktionellen autonomen Volumens unabhängig von seiner intrathyreoidalen Verteilung

D. Emrich, U. Erlenmaier, M. Pohl, H. Luig

Unterscheiden sich hyperthyreote funktionelle Autonomien von euthyreoten hinsichtlich ihres TcTU's oder ihres autonomen Volumens? Diskriminanzanalyse von funktionellen Schilddrüsenautonomien bei endogener TSH-Suppression E. Werner, J. Rendl, M. Scheubeck, W. Börner

2.2 Beobachtung und Behandlung von Schilddrüsenerkrankungen Jodausscheidung, Patientencompliance und Änderungen im Schilddrüsenvolumen unter Strumatherapie J. Rendl, S. Seybold, W. Börner

Strumarezidivprophylaxe mit und ohne L-Thyroxin bei 104 Patienten; retrospektive Langzeitbeobachtung über 6,4 Jahre A. H. Rzepka, Th. Olbricht, D. Reinwein

Klassifikation von 445 Patienten mit Hypothyreose in einem Jodmangelgebiet

Ch. Jaspers, A. Kirbas, D. Reinwein

Schilddrüsenvolumen und Wachstumshormonsubstitution bei Erwachsenen mit Hypophysenvorderlappeninsuffizienz

P. Kann, B. Piepkorn, G. Kahaly, J. Beyer

Hypophysäre Schilddrüsenhormonresistenz mit neonataler

Hyperthyreose

M. Klett, D. Schönberg

Fehldiagnose einer Autonomie bei Patienten mit chronischer Autoimmunthyreoiditis M. Grußendorf

Untersuchungen zur körperlichen Leistungsfähigkeit bei

Schilddrüsenfunktionsstörung

J. Hellermann, G. Kahaly

\subsection{Schilddrüsenmalignität}

Die Wertigkeit der ${ }^{99 \mathrm{~m}} \mathrm{Tc}-\mathrm{MIBI}$-Szintigraphie in der Rezidivdiagnostik bei differenzierten Schilddrüsenkarzinomen

H. Elser, I. Mattern-Alvarez, P. Georgi 
Intraoperative Rezidivlokalisation von medullären

Schilddrüsenkarzinomen mittels einer Gammasonde:

erste klinische Ergebnisse

508

S. Adams, R. P. Baum, H. J. C. Wenisch, G. Herrmann, A. Niesen,

A. Encke, G. Hör

Wertigkeit verschiedener diagnostischer Parameter bei der Erkennung von Frührezidiven bei Patienten mit Schilddrüsenkarzinomen

S. Weiß, E. Henke, R. Schnorrfeil

Schilddrüsenkarzinom und Hyperthyreose

518

M. Auersperg, M. Hočevar, M. Us-Krašovec, T. Movrin, B. Novak

Hyperthyreose und Schilddrüsenkarzinom:

Ergebnisse einer regionalen Erhebung

J. Winter, W. Back, S. Mehl, R. Paschke, M. Knoll, R. Frentzel-Beyme

Einfluß des TNM-Stadiums und weiterer Parameter auf die Prognose von Schilddrüsentumoren

F. Grünwald, P. Ullmann, R. Fimmers, A. Hotze, H.-J. Biersack

Das pT4-Stadium beim papillären Schilddrüsenkarzinom:

eine eigene Prognoseentität?

O. Gimm, G. F. W. Scheumann, G. Wegener, H. Dralle

3 Empfehlungen für die Praxis

Neueste Fortschritte in der Schilddrüsendiagnostik:

In-vitro-Tests - methodische und klinische Aspekte

H. Meinhold, W. Becker

Empfehlungen für die Schilddrüsendiagnostik und die Diagnose von

Schilddrüsenkrankheiten - Zusammenfassung

G. Hintze

Morbus Basedow: welche Therapie für den individuellen Patienten?

R. Ziegler, Ch. Herfarth, P. Georgi

Verzeichnis der erstgenannten Autoren

Sachverzeichnis 
